ARTICLE

\title{
Genome of Tripterygium wilfordii and identification of cytochrome P450 involved in triptolide biosynthesis
}

Lichan Tu (1) 1,2,9, Ping Su 3,8,9, Zhongren Zhang (1) 4,9, Linhui Gao1, Jiadian Wang1, Tianyuan Hu (1) 1, Jiawei Zhou?', Yifeng Zhang ${ }^{1}$, Yujun Zhao ${ }^{3}$, Yuan Liu', Yadi Song ${ }^{1}$, Yuru Tong ${ }^{3}$, Yun Lu' ${ }^{1}$ Jian Yang ${ }^{3}$, Cao Xu (1) ${ }^{5}$, Meirong Jia ${ }^{6}$, Reuben J. Peters (iD) ${ }^{6}$, Luqi Huang ${ }^{3 凶} \&$ Wei Gao (i) $1,2,7 凶$

Triptolide is a trace natural product of Tripterygium wilfordii. It has antitumor activities, particularly against pancreatic cancer cells. Identification of genes and elucidation of the biosynthetic pathway leading to triptolide are the prerequisite for heterologous bioproduction. Here, we report a reference-grade genome of $T$. wilfordii with a contig N50 of $4.36 \mathrm{Mb}$. We show that copy numbers of triptolide biosynthetic pathway genes are impacted by a recent whole-genome triplication event. We further integrate genomic, transcriptomic, and metabolomic data to map a gene-to-metabolite network. This leads to the identification of a cytochrome P450 (CYP728B70) that can catalyze oxidation of a methyl to the acid moiety of dehydroabietic acid in triptolide biosynthesis. We think the genomic resource and the candidate genes reported here set the foundation to fully reveal triptolide biosynthetic pathway and consequently the heterologous bioproduction.

\footnotetext{
${ }^{1}$ School of Traditional Chinese Medicine, Capital Medical University, Beijing, China. ${ }^{2}$ School of Pharmaceutical Sciences, Capital Medical University, Beijing, China. ${ }^{3}$ State Key Laboratory Breeding Base of Dao-di Herbs, National Resource Center for Chinese Materia Medica, China Academy of Chinese Medical Sciences, Beijing, China. ${ }^{4}$ Novogene Bioinformatics Institute, Beijing, China. ${ }^{5}$ University of Chinese Academy of Sciences, Beijing, China. ${ }^{6}$ Roy J. Carver Department of Biochemistry, Biophysics \& Molecular Biology, lowa State University, Ames, IA, USA. ${ }^{7}$ Advanced Innovation Center for Human Brain Protection, Capital Medical University, Beijing, China. ${ }^{8}$ Present address: Department of Chemistry, The Scripps Research Institute, Jupiter, Florida, USA.

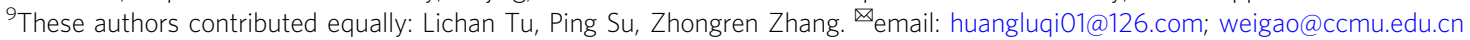


T ripterygium wilfordii, a perennial twining shrub of the Celastrales, has been used medicinally for centuries, mainly to treat rheumatoid arthritis ${ }^{1}$. It has been known to be a rich source of specialized metabolites. Two of these (triptolide and celastrol) are among five natural products highlighted for their great potential to be developed into pharmaceuticals ${ }^{2}$. Indeed, triptolide has been demonstrated to possess important therapeutic potential with anti-inflammatory, immunosuppressive, and antitumor activities, as well as to exhibit potentially medically relevant activity for central nervous system diseases (e.g. Parkinson's and Alzheimer's diseases) ${ }^{2-8}$. Moreover, several derivatives of triptolide have undergone clinical trials $s^{9-11}$.

Currently, triptolide only can be extracted from $T$. wilfordii with extremely low yields, ranging from $0.0001 \%$ to $0.002 \%$ of dry weight biomass ${ }^{12,13}$, and the plant cannot be cultivated at a large scale as contamination with its pollen renders honey poisonous, which has frequently causes poisoning events in areas where this medicinal plant is cultivated. Although significant efforts have been devoted to improving chemical synthesis, current routes are limited to yields of less than $1.64 \%$ due to the structural and stereochemical complexity of triptolide ${ }^{14-17}$. Accordingly, further investigation of its pharmaceutical utility is severely limited by a shortage of supply. While suspension cultures, tissue cultures, and adventitious root cultures have been investigated as alternative sources of this bioactive diterpenoid ${ }^{18-22}$, a more promising approach to obtaining such structurally complex natural products is metabolic engineering. This can be attempted in the native plant or accomplished via a synthetic biology strategy, involving reconstitution in a suitably engineered microbial chassis organism, which can establish a sustainable and reliable means of production ${ }^{23-26}$. However, this latter approach requires elucidation of the relevant biosynthetic pathway.

Triptolide is an abietane-type diterpenoid, produced via initial cyclization of $(E, E, E)$-geranylgeranyl diphosphate (GGPP) to copalyl diphosphate (CPP), with subsequent cyclization to the abietane-type diene olefin miltiradiene $e^{27,28}$. The 1,4-diene arrangement of the miltiradiene $\mathrm{C}$ ring leaves this poised for aromatization ${ }^{29}$, which most likely occurs spontaneously ${ }^{30}$. Along with conversion of carbon-18 (C-18) from a methyl to carboxylic acid, this forms dehydroabietic acid, followed by oxidative 1,2migration of C-18 (from C- $4 \rightarrow \mathrm{C}-3$ ) and further transformation to the phenolic triepoxide triptolide ${ }^{27,28}$. At present, elucidation of the triptolide pathway relies on transcriptomes, which has only led to identification of the relevant diterpene synthases, namely the relevant CPP synthase (CPS1) and miltiradiene synthase (MS) ${ }^{28,31}$, with subsequently acting enzymes, such as cytochrome P450s (CYPs), involved in further biosynthesis of the highly functionalized triptolide remaining enigmatic. CYPs form the largest family of enzymes in plants, playing manifold roles in their complex metabolism ${ }^{32}$. Indeed, there are still CYP families, defined as phylogenetic clades with $<40 \%$ amino acid sequence identity between them ${ }^{32}$, whose function remains unknown, with no biochemical activity yet assigned to any member ${ }^{32}$. Recently, whole-genome sequencing provides a comprehensive genetic resource and has become a practical approach to not only elucidation of natural product biosynthetic pathways, but also insight into their evolution, as well as improvement of their production 33,34 . Nevertheless, this must be coupled to other information directed more specifically at the natural product of interest.

Here we first present a high-quality reference-grade genome of T. wilfordii and show that duplications of triptolide biosynthetic pathway genes are almost all generated by a recent whole-genome triplication event. We then map a gene-to-metabolite network by integrating genomic, transcriptomic, and metabolomic data. Next, we combine the synthetic biology tools, RNAi knock-down, and overexpression to identify a cytochrome P450 (CYP728B70) that can catalyze oxidation of $\mathrm{C}-18$ from a methyl to the acid moiety of dehydroabietic acid in triptolide biosynthesis. This work provides the genomic resource and the candidate genes that may contribute to fully elucidation of the triptolide biosynthetic pathway and consequently lead to heterologous bioproduction.

\section{Results}

Genome assembly and annotation. Based on the k-mer distribution analysis, we estimated the genome size of $T$. wilfordii to be $\sim 365.95 \mathrm{Mb}$ with a high level of heterozygosity $(1.95 \%)$ and repetition $(48.87 \%)$, indicating the genome assembly was complicated (Supplementary Fig. 1 and Supplementary Table 1). The genome of T. wilfordii was sequenced using PacBio (read length of $60 \mathrm{~kb}, \sim 207.10 \times$ coverage) and 10X Genomics ( 327.23× coverage) (Supplementary Table 2). The total length of the final assembly was $348.38 \mathrm{Mb}$ with 467 contigs and a contig N50 of $4.36 \mathrm{Mb}$ (Supplementary Table 3). Assessment of the completeness of the genome assembly with CEGMA ${ }^{35}$ indicated $96.77 \%$ coverage of the conserved core eukaryotic genes (Supplementary Table 5), and $\mathrm{BUSCO}^{36}$ results indicated that the genome was 95.10\% complete (Supplementary Table 6). Additionally, 97.06\% of the transcriptome can be mapped back to the assembly, further supporting a high level of genome coverage (Supplementary Table 7 and Supplementary Note 1).

The T. wilfordii assembly was further refined using highthroughput chromosome conformation capture (Hi-C) data, comprised of 321 scaffolds with a scaffold N50 of $13.52 \mathrm{Mb}$ (Table 1). As a result, $315.08 \mathrm{Mb}$ of the assembly and $99.92 \%$ of the genes were distributed across 23 chromosome-level pseudomolecules (Fig. 1a, Table 1 and Supplementary Data 1).

We were able to annotate 28,321 protein-coding genes, with an average sequence length of $3338 \mathrm{bp}$, similar to those of other reported plants (Supplementary Tables 8 and 9). On average, each predicted gene contains 5.44 exons with an average sequence length of $228 \mathrm{bp}$. A total of $182.52 \mathrm{Mb}$ of repetitive elements occupying $52.36 \%$ of the T. wilfordii genome were annotated (Supplementary Fig. 2 and Supplementary Note 2). The majority of the repeats are long terminal repeats (LTRs) $(34.26 \%$ of the genome; Supplementary Table 10). Approximately $99.6 \%$ of the genes were functionally annotated by similarity searches against homologous sequences and protein domains (Supplementary Table 11). In addition, we identified noncoding RNA (ncRNA) genes, including 2,563 rRNA, 407 tRNA, 373 miRNA, and 892 snRNA genes (Supplementary Table 12). These results further support the completeness of our $T$. wilfordii genome

\begin{tabular}{|lll|}
\hline $\begin{array}{l}\text { Table 1 Summary of T. wilfordii genome assembly and } \\
\text { annotation. }\end{array}$ & Size \\
\hline \multicolumn{2}{l}{ Number } & \\
\hline Genome assembly & & $348.38 \mathrm{Mb}$ \\
Total contigs & 467 & $4.36 \mathrm{Mb}$ \\
Contig N50 & 15 & $265 \mathrm{~kb}$ \\
Contig N90 & 137 & $348.53 \mathrm{Mb}$ \\
Total scaffolds & 321 & $13.52 \mathrm{Mb}$ \\
Scaffold N50 & 12 & $10.83 \mathrm{Mb}$ \\
Scaffold N90 & 23 & $315.08 \mathrm{Mb}$ \\
Pseudochromosomes & 23 & $182.52 \mathrm{Mb}$ \\
Genome annotation & & $1.12 \mathrm{Mb}$ \\
Repetitive sequences & $52.36 \%$ & $109.30 \mathrm{Mb}$ \\
Noncoding RNAs & 4235 & $109.24 \mathrm{Mb}$ \\
Protein-coding genes & 28,321 & \\
Genes in pseudochromosomes & $28,297(99.92 \%)$ & \\
\hline
\end{tabular}


a

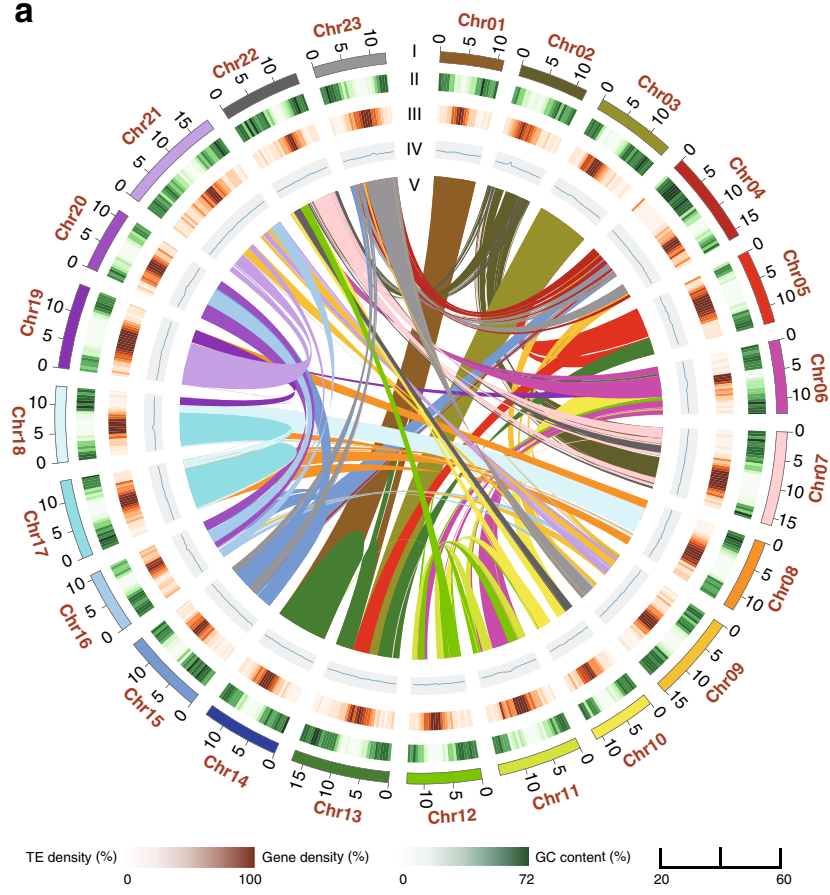

b

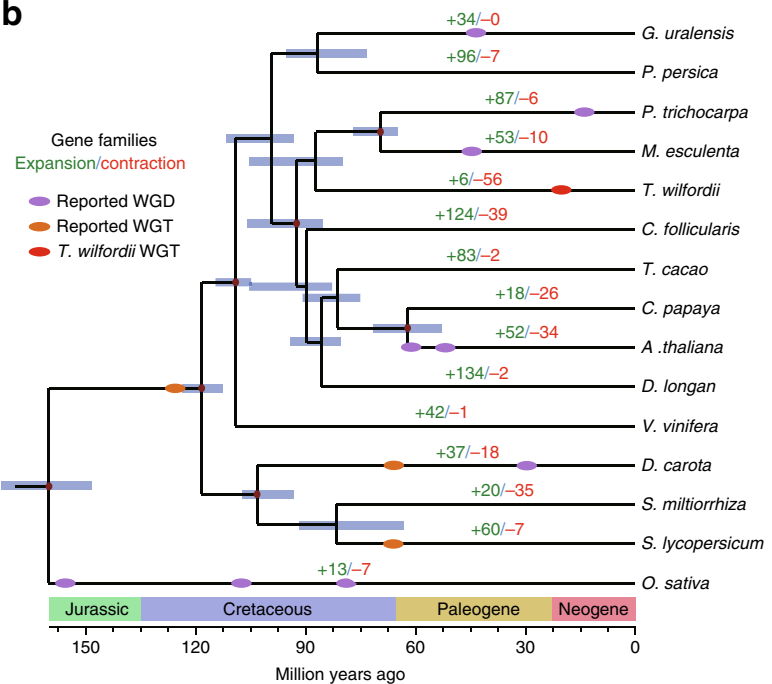

C
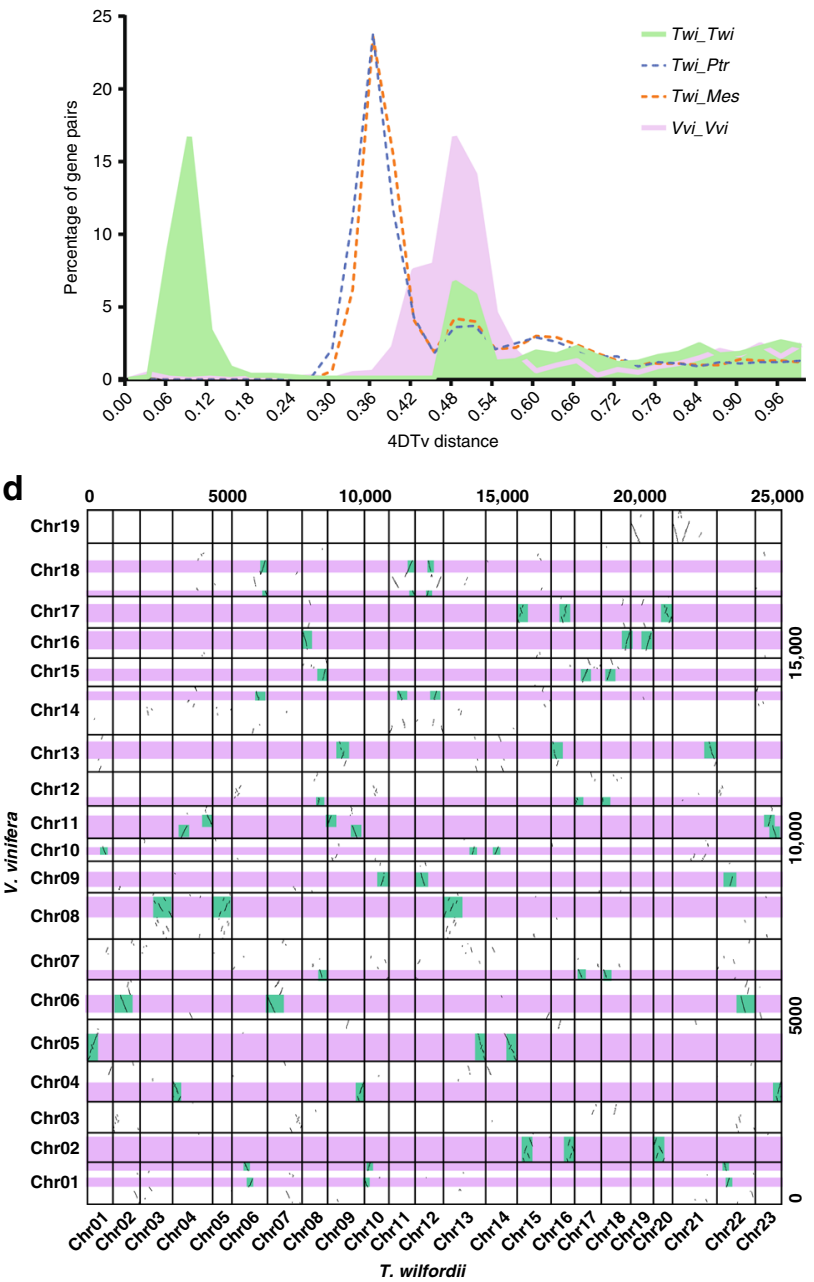

e

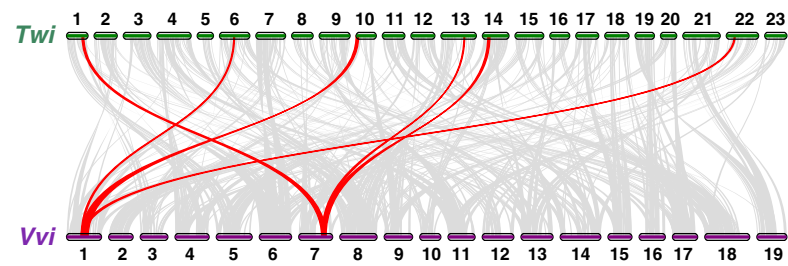

Fig. 1 Genome evolution of T. wilfordii. a Distribution of T. wilfordii genomic features. (I) Circular representation of the pseudomolecule. (II-IV) gene density ( $500 \mathrm{~kb}$ window), percentage of repeats ( $500 \mathrm{~kb}$ window), and GC content (500 kb window). (V) Each linking line in the center of the circle connects a pair of homologous genes. b Inferred phylogenetic tree with 514 single-copy genes of 15 plant species. Gene family expansions are indicated in green, and gene family contractions are indicated in red. The timing of whole-genome duplication (WGD) and the timing of whole-genome triplication (WGT) are superimposed on the tree. Divergence times are estimated by Maximum Likelihood (PAML). c Distribution of 4DTv shown in colored lines as indicated. d Syntenic dot plots show a 3-1 chromosomal relationship between $T$. wilfordii genome and $V$. vinifera genome. e Macrosynteny between T. wilfordii and V. vinifera karyotypes. Source data are provided as a Source Data file.

sequence and a schematic representation of the genome is given in Fig. 1a.

Genome evolution contributed to formation of triptolide. To investigate the evolution of T. wilfordii, we constructed a phylogenetic tree and estimated the divergence times of 15 plant species using 514 single-copy genes (Fig. 1b, Supplementary Fig. 4 and Supplementary Note 3). Phylogenomic analysis showed that T. wilfordii was most related to the ancestor of Manihot esculenta and Populus trichocarpa, with an estimated divergence time of 87.1 million years ago (MYA). Gene family expansion and contraction was examined using CAFÉ (Fig. 1b and Supplementary Note 3). Among the T. wilfordii, P. trichocarpa, $M$. esculenta, and Cephalotus follicularis gene families, a total of 951 genes appeared unique to T. wilfordii (Supplementary Fig. 5). Interestingly, Gene Ontology (GO) and Kyoto Encyclopedia of Genes and Genomes (KEGG) analyses found these T. wilfordiispecific genes were particularly enriched in the terms terpene synthase, oxidation-reduction process, and plant-pathogen interaction (Supplementary Tables 13 and 14).

The distribution of 4DTv (fourfold degenerate synonymous sites of the third codons) of all gene pairs found in each segment 
showed two peaks at approximately 0.09 and 0.48 in the T. wilfordii genome. The first peak at approximately 0.48 revealed the core eudicot $\gamma$ triplication event, and the second peak at approximately 0.09 indicated that $T$. wilfordii underwent another whole-genome multiplication event after diverging from $P$. trichocarpa and M. esculenta (Fig. 1c). We further compared T. wilfordii genome and Vitis vinifera genome. $77 \%$ of T. wilfordii gene models are in syntenic blocks corresponding to one $V$. vinifera region, covering $83 \%$ of the $V$. vinifera gene space, among which $35 \%$ have three orthologous regions in T. wilfordii, $31 \%$ have two, and $15 \%$ have one (Supplementary Fig. 6). Intergenomic co-linearity analysis was consistent with both the $\gamma$-event and another, more specific WGT event for T. wilfordii, as indicated by a 1:3 syntenic relationship between $T$. wilfordii and $V$. vinifera (Fig. 1d, e). The recent WGT event was dated to approximately $21 \pm 6 \mathrm{MYA}$, as indicated by the distribution of synonymous substitutions per synonymous site (KS) of syntenic genes in T. wilfordii (Supplementary Fig. 7). The recent WGT event occurred in the Paleogene (65-23.3 MYA) to Neogene (23.3-1.64 MYA) period, which may have enabled T. wilfordii to cope better with the markedly changed environment by functional redundancy, mutational robustness, increased evolution rate, and adaptation ${ }^{37}$.

Gene families that may be involved in terpenoid (e.g., triptolide in T. wilfordii) biosynthesis were identified in the fifteen reported plant species. The results showed that the copy number of these gene families varied among all examined plant species, with those encoding DXS, GGPPS, TPS and CYP exhibiting particularly strong variation (Supplementary Table 18). To further investigate the role of WGT events on triptolide biosynthesis, we carried out phylogenetic analysis of the gene families potentially involved in this pathway, specifically $K s$ calculations for each duplicated gene pair, including those from upstream isoprenoid metabolism (i.e., ACAT, CMK, DXS, FPS, GPS, GGPPS, HDR, HDS, HMGR, HMGS, IDI, MCT, MVK, MVD, PMK) and those more specific to triptolide (i.e., $C P S$ and $M S$ ) (Supplementary Fig. 8 and Supplementary Table 19). We found that duplications of these genes were almost all generated by the recent WGT event (i.e., ACAT, CMK, GPS, HDR, HMGS, IDI, MVK, MVD, PMK, CPS and $M S$ ) (Supplementary Fig. 9), suggesting that the recent WGT event were important to the evolution of triptolide biosynthesis in T. wilfordii.

Notably it was found that the TwCPS1 and TwMS genes already known to be involved in triptolide biosynthesis are adjacent to each other in the T. wilfordii genome (Supplementary Fig. 10), which is similar to previously identified biosynthetic gene clusters in other plant genomes ${ }^{38-40}$. However, while the relevant region on chromosome 21 also contains a CYP (TW023804.1) that exhibits a similar expression pattern, this is sufficiently distant, including a number of intervening genes clearly unrelated to triptolide biosynthesis, to leave its relevance unclear. Similarly, while there are several nearby transcription factors (TFs), as well as another CPS, these are unlikely to play a role in the production of triptolide.

Integrated transcriptome and metabolome analysis. To gain further insights into triptolide biosynthesis, as well as the organization and regulation of the triptolide pathway, suspension cells induced by methyl jasmonate (MeJA) and seven different tissues were used as the source of transcripts and metabolites (Supplementary Notes 4 and 5). The results showed that triptolide levels in MeJA-induced cells were 3.6-fold higher (relative to control cultures) after $360 \mathrm{~h}$, while the levels of triptophenolide were even higher, 55-fold (Fig. 2). More detailed analysis was focused on a final set of 142 peaks with a mass ratio of 295-400, the expected range for diterpenoids (Supplementary Figs. 11-14 and Supplementary Note 7). For example, the content of these potentially triptolide-related metabolites was highest in the root bark (Supplementary Figs. 15 and 16). Data sets for these accumulated metabolites and gene expression (including all CYPs, TwCPS1 and TwMS) were separately normalized, and correlation network analysis was used to establish gene-to-metabolite coregulation patterns ${ }^{41}$ in suspension cells and the various tissues, respectively. The Pearson correlation coefficient between each set of variables (either metabolite or gene) was calculated, including all conditions and time points (Supplementary Data 3 and 4). The correlation network was analyzed by Cytoscape (version 3.6.1), using a correlation coefficient $>0.7$ as the cutoff (Supplementary Figs. 19 and 20). The utility of the gene-to-metabolite network was verified by the observation that the key genes TwCPS1 and TwMS were both strongly associated with triptolide and triptophenolide. Accordingly, we proceeded under the assumption that the CYP catalyzing the next step to form dehydroabietic acid should similarly be present among such strongly related genes. Indeed, a total of 97 CYP genes, of which 57 genes were reasonably well-expressed-i.e., with RPKM $>1$ (Supplementary Fig. 21 and Supplementary Data 6) -were strongly associated with triptolide in the network. These then are potentially involved in triptolide biosynthesis.

Identification of specific CYP genes in $T$. wilfordii. So far, triptolide is found only in Tripterygium, indicating that some of the CYPs involved in the biosynthesis of triptolide may be specific to T. wilfordii. In order to identify such species-specific CYPs, we annotated a total of 2335 CYP genes in Arabidopsis thaliana, Daucus carota, Glycyrrhiza uralensis, Prunus persica, P. trichocarpa, Solanum lycopersicum, Salvia miltiorrhiza and T. wilfordii (Supplementary Table 18). We constructed a phylogenetic tree from amino acid sequence alignment of all these CYPs and identified T. wilfordii specific CYPs using a cutoff of 55\% identity, which indicates separate sub-family assignment ${ }^{42}$. This revealed 22 T. wilfordii-specific CYP genes (Supplementary Data 5). Interestingly, expression levels of six of these CYPs were significantly increased by MeJA induction, and most of these were highly expressed in root bark in which the terpene synthase genes were enriched and most of them were highly expressed (Supplementary Figs. 17, 18 and 22), leading to strong correlation with triptolide and/or triptophenolide.

Functional identification of CYP728B70. Among CYP genes correlated with triptolide in the gene-to-metabolite network, as well as those specific to T. wilfordii, there were 13 found to express differentially between MeJA-induced and control cells at $4,12,24,48$, and $72 \mathrm{~h}$, and/or express differentially between root bark and other tissues (e.g., flower, stem bark, peeled stem, and leaf), and also exhibit highly similar expression patterns as TwCPS1 and TwMS, including high expression levels in root bark (Fig. 3a, Supplementary Fig. 18 and Supplementary Data 6). Among these candidates, TW016590.1 was already previously identified as ent-kaurene oxidase ${ }^{43}$, TW012756.1 was annotated as trans-cinnamate 4-monooxygenase, which is not likely to be involved in terpenoid biosynthesis, while TW017699.1 and TW013472.1 appeared to arise from a relatively recent tandem gene duplication event as their amino acid sequences are $97 \%$ identical (Supplementary Data 2), and we chose to only target TW017699.1 directly. Accordingly, we settled on a total of 10 candidates for the follow-up RNAi studies, which were carried out with suspension cell cultures to provide more direct evidence for a role in triptolide biosynthesis for these 10 candidates 


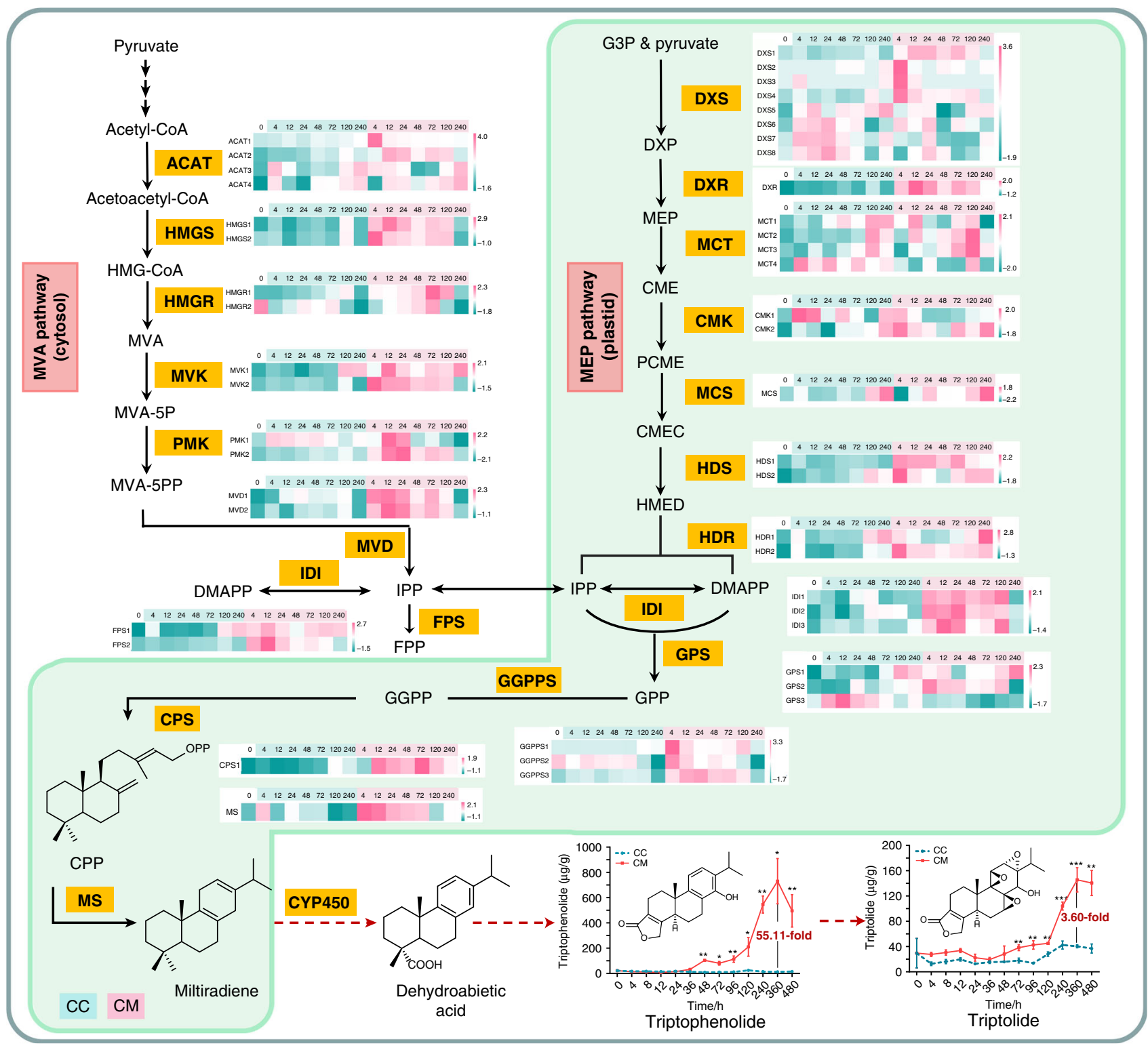

Fig. 2 Comparative transcriptomic analysis of genes involved in the triptolide biosynthetic pathway. Suspension cells of $T$. wilfordii were treated with methyl jasmonate and DMSO. 0, 4, 12, 24, 48, 72, 120, 240 represent the time points of each sample, CC means the control group of cells, and CM means the group of MJ-treated cells. Heat maps of these genes were plotted using MeV software (version 4.9.0). Error bars, mean \pm SD ( $n=3$ biologically independent samples; ${ }^{\star} P<0.05,{ }^{\star \star} P<0.01,{ }^{\star \star \star} P<0.001$ by 2 -sided Student's $t$ test). Source data are provided as a Source Data file.

(Supplementary Table 20). In 4 RNAi-lines (CYP728B70, TW011445.1, TW012149.1, TW006625.1), the transcript levels of the targeted gene and triptolide accumulation were decreased compared to those in control cultures transformed with an empty vector (Fig. 3b). These four CYPs were co-expressed with the cytochrome $\mathrm{P} 450$ reductase $3(\mathrm{CPR} 3)^{43}$ in yeast also engineered to produce miltiradiene, as previously described ${ }^{44}$. Gratifyingly, with CYP728B70, which was strongly associated with triptolide in the gene-metabolite network, this led to the formation of four compounds, dehydroabietic acid (abieta-8,11,13-trien-18-oic acid, 1), miltiradienoic acid (abieta-8,12-dien-18-oic acid, 2), dehydroabietinol (abieta-8,11,13-trien-18-ol, 3) and miltiradienol (abieta-8,12-dien-18-ol, 4), whose structures were identified after purification by ${ }^{1} \mathrm{H}$ NMR and ${ }^{13} \mathrm{C}$ NMR (Fig. 3c, Supplementary Figs. 24-32 and Supplementary Note 6). To further verify such activity, in vivo assays also were performed, that is, substrate feeding of cultures expressing CYP728B70 in the yeast WAT11 strain that also expresses an Arabidopsis $\mathrm{CPR}^{45}$. Compounds 1-4 were detected when feeding miltiradiene (abieta-8,12-diene), while only the derived compounds 1 and $\mathbf{3}$ were detected when feeding abieta-8,11,13-triene. Similarly, compounds 1 and 2 were detected when feeding compound 4 (miltiradienol), while only compound $\mathbf{1}$ was detected when feeding compound 3 (dehydroabietinol) (Fig. 3d, e). Given that aromatization of the miltiradiene $\mathrm{C}$ ring to form abietatriene most likely occurs spontaneously, these results indicate that TwCYP728B70 catalyzes consecutive oxidations at C-18 of miltiradiene or abietatriene to form the corresponding alcohol and acid derivatives. Although the expected aldehyde intermediate is not observed, this almost certainly also is formed between the alcohol and acid final products.

To further explore the role of CYP728B70 in triptolide biosynthesis, overexpression was employed. This led to a significant increase in transcript level, albeit only by $\sim 1.6$-fold 
a

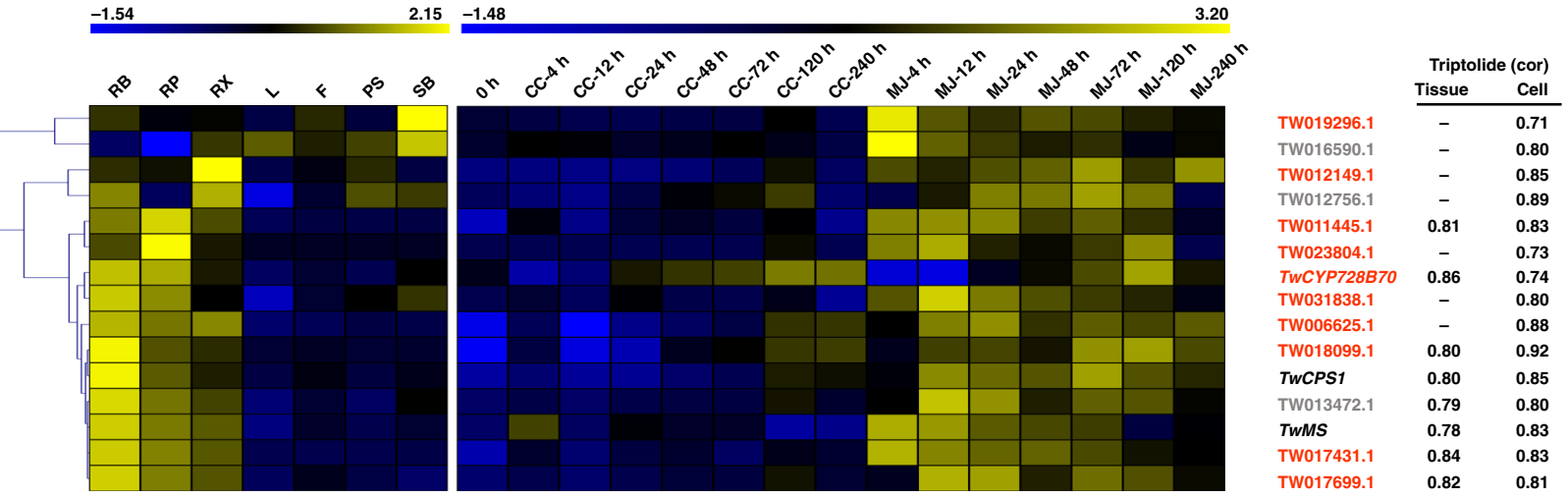

b
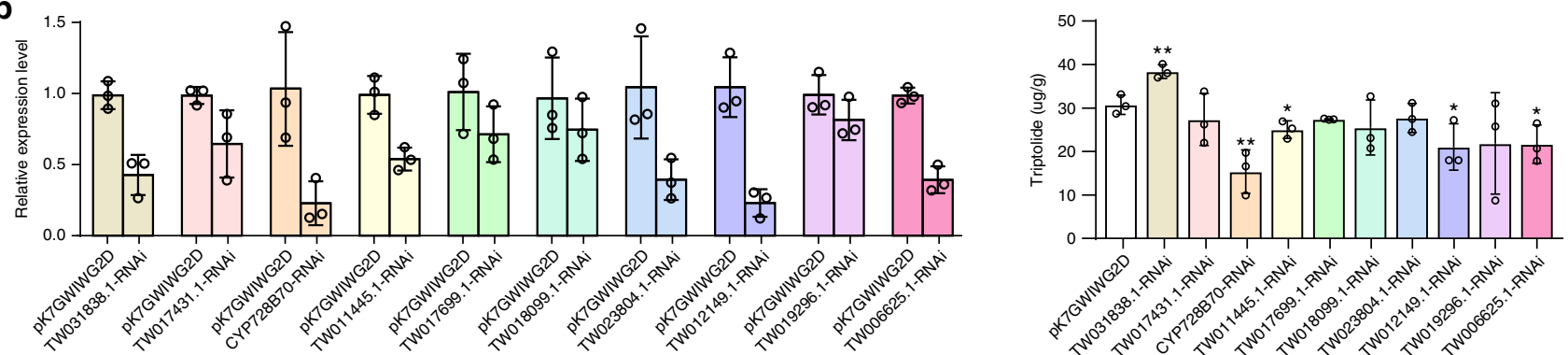

C

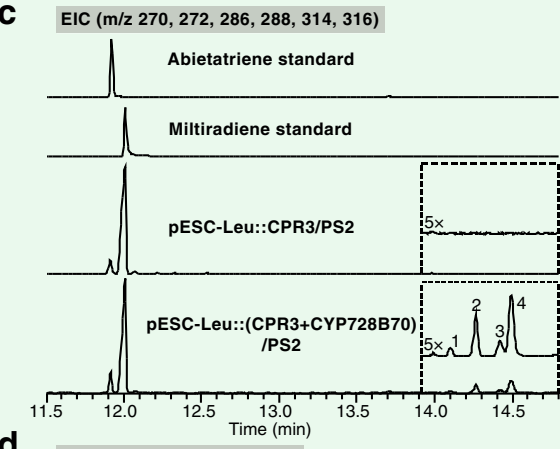

d

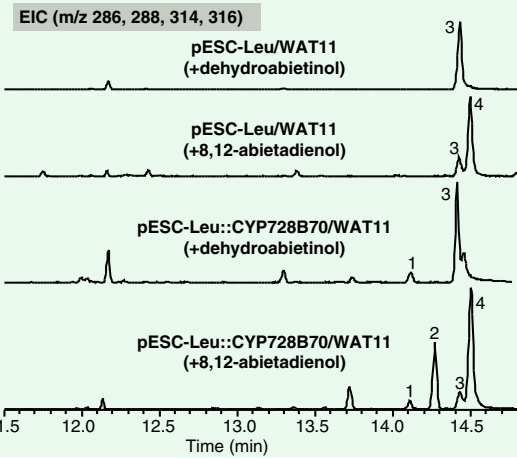

e EIC $(m / z 270,272,286,288,314,316)$

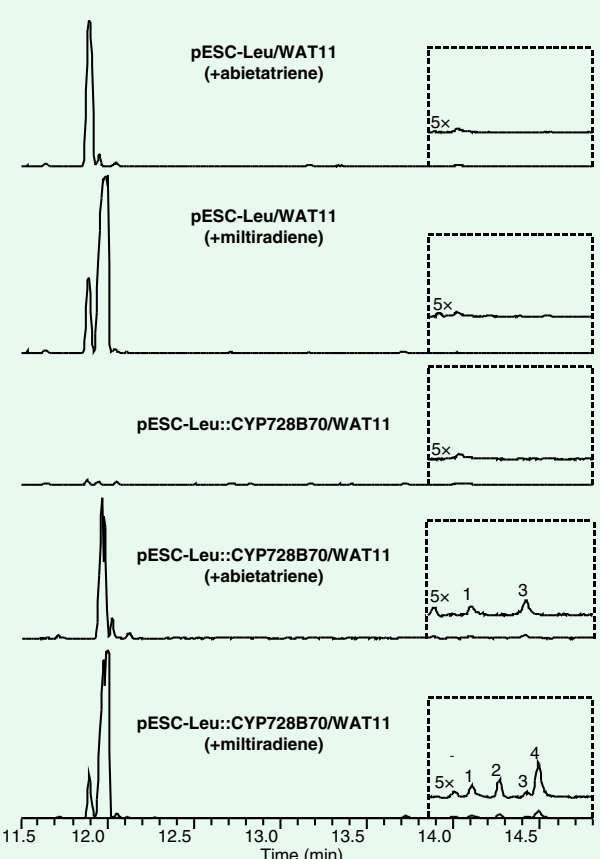

f
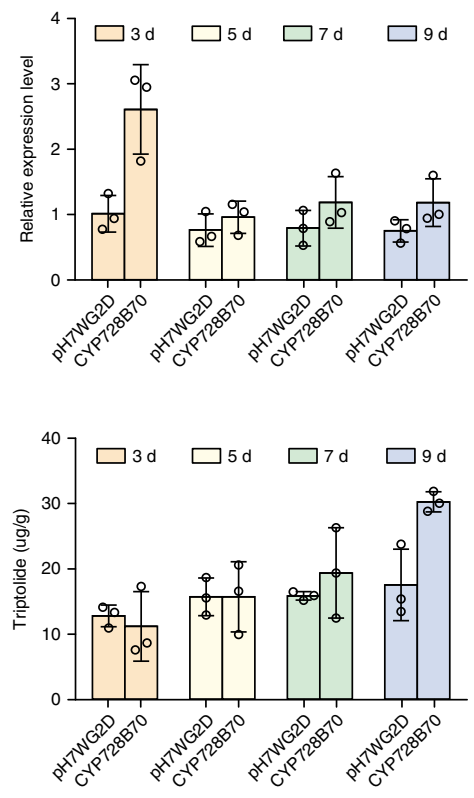

Fig. 3 CYP gene screening and functional identification of CYP728B70. a Hierarchical clustering of RNA-Seq expression data after filtering by expression level. b Relative expression of 10 candidate CYPs and triptolide concentration in RNAi suspension cells. c Co-expression of CYP728B70 and TwCPR3 in miltiradiene engineering yeast. $\mathbf{d}$ In vivo transformation of authentic diterpene alcohol substrates to the corresponding alcohols and acids in cultures of yeast cells expressing CYP728B70. e In vivo transformation of authentic diterpene substrates to the corresponding alcohols and acids in cultures of yeast cells expressing CYP728B70. f Relative expression of CYP728B70, as well as triptolide concentration in CYP728B70-overexpressing suspension cells on the 3rd, 5th, 7th, 9th day. Error bars, mean \pm SD ( $n=3$ biologically independent samples; ${ }^{\star} P<0.05$, ${ }^{\star} P<0.01$ by 2 -sided Student's $t$ test). Source data underlying Figs. 3a, b, f are provided as a Source Data file.

compared with the control cultures transformed with the empty vector, on the 3rd day, and it then decreased but was still slightly higher than in the control cultures at later time points. Nevertheless, elevated levels of triptolide in the overexpression line relative to those in the control cultures were evident on the 9th day, reaching just over $30.5 \mu \mathrm{g} \mathrm{g}^{-1}$ in the overexpression line, representing an $\sim 70 \%$ increase (Fig. $3 \mathrm{f}$ ). This not only further supports a role for CYP720B70 in triptolide biosynthesis, but also showcases its potential utility for improving production of this valuable diterpenoid in $T$. wilfordii. 
To explore the potential functions of the other 9 candidate CYPs, in vitro enzymatic activity assays were performed using the available dehydroabietic acid, triptinin $B$, triptophenolide and triptoquinonide, all of which are likely intermediates in triptolide biosynthesis (Supplementary Note 7). However, no new compounds were observed, indicating that these CYPs could not catalyze transformation of these intermediates, but does not rule out a role for them in triptolide biosynthesis (i.e., in mediating reactions involving other intermediates) (Supplementary Fig. 33).

\section{Discussion}

Triptolide, a structurally complex phenolic diterpene triepoxide of $T$. wilfordii, has potent antitumor and immunosuppressive activities. Research focusing on its biosynthetic pathway has been stymied, in part, by the absence of a comprehensive genetic accounting. The high-quality reference-grade genome of T. wilfordii reported here thus provides a valuable genomic resource for investigation of triptolide biosynthesis, and is, to the best of our knowledge, the first genomic sequence for a plant of the order Celastrales. Accordingly, it further represents a cornerstone for evolutionary phylogenomic studies of not only $T$. wilfordii, but the Celastrales order more generally.

Previous investigations of triptolide biosynthesis relied on transcriptome data, and only identified the relevant diterpene synthases. Herein, we integrated genomic, transcriptomic, and metabolomic data to map a gene-to-metabolite network, screening out 57 CYP genes which were potentially involved in triptolide biosynthesis. Then we combined the co-expression patterns, tissue-specificity and inducibility of these candidate genes as well as $T$. wilfordii-specific CYP genes for further investigations, resulting in a total of 10 candidates. Through the above analysis, we identified 4 CYP genes involved in triptolide biosynthesis, as RNAi knock-down of these led to decreased accumulation of this natural product. Moreover, we successfully characterized the role of CYP728B70, which is responsible for oxidation of C-18 from a methyl to the acid moiety of dehydroabietic acid, in triptolide biosynthesis. As demonstrated here by substrate feeding studies, CYP728B70 exhibits multiple/sequential reactivity in carrying out this transformation in triptolide biosynthesis. Consistent with the limited accumulation of triptolide in T. wilfordii, CYP728B70 exhibits low transcript levels, although it is highest in the root bark where this diterpenoid is primarily found. Notably, overexpression of CYP728B70 in plant cell cultures led to a significant $(\sim 70 \%)$ increase in triptolide levels relative to control cultures, indicating that CYP728B70 activity limits triptolide biosynthesis, and demonstrating the utility of the results reported here for improving the yield of this potential pharmaceutical agent.

Perhaps not surprisingly given that CYPs form the largest family of enzymes, there are still CYP families that are completely uncharacterized. Indeed, this included the CYP728 family, which was first observed in Amborella, although it has been lost in Arabidopsis (Supplementary Fig. 35), and no function has been previously reported for any member of this CYP family ${ }^{32}$. Hence, CYP728B70 appears to be the only one member of this CYP family whose catalytic activity has been identified, and its role in diterpenoid metabolism immediately suggests similar function for other family members. Also, the stepwise carboxylation reaction observed for CYP728B70 is catalytically highly similar to the CYP720B family in conifers ${ }^{46,47}$ that even forms some common products, thus showcasing an intriguing case of independent evolution of these functions in distant plant species. In addition, the other 3 CYP genes that affect the biosynthesis of triptolide in the results of RNAi experiment are from T. wilfordii-specific CYP (sub-)families. Although we have not identified their catalytic functions, such biochemical characterization will then provide similar insight.

Although some steps in the triptolide biosynthetic pathway still remain unknown, we have provided many promising candidates, including CYP genes and metabolites that might constitute yet-unknown intermediates or side products from triptolide biosynthesis. We have also provided many potential TFs that are most likely involved in the regulation of the biosynthesis of triptolide (Supplementary Fig. 34, Supplementary Data 8-13 and Supplementary Note 8). Of particular note here is the identification of roles for CYPs from (sub-)families that are specific to T. wilfordii, as much of the reported work in such characterization of CYP function in plant natural products biosynthesis has to some extent relied on homology at least at the family, if not subfamily, level.

In addition to our identification of the role of CYP728B70, we used this to engineer yeast for the production of dehydroabietic acid (Fig. 4). This lays the foundation for identification of genes encoding subsequently acting enzymes, and will be invaluable in future investigations of triptolide biosynthesis.

In conclusion, while it is difficult to resolve the biosynthetic pathways of the complex natural products in non-model systems, we have demonstrated here the utility of the multi-omics data, as well as co-expression patterns, tissue-specificity, and inducibility of candidate genes will contribute for such investigations. Interestingly, while genome sequencing found pairing of the genes for the consecutively acting TwCPS1 and TwMS can initiate triptolide biosynthesis, these do not appear to have be co-clustered with genes for later acting enzymes. Nevertheless, the genome sequence reported here provides a comprehensive genetic inventory, which was coupled with the observation that triptolide production is tissue-specific and affected by elicitors, much as found with other plant natural products ${ }^{48}$, to generate gene-to-metabolite networks that can be productively mined. Notably, this approach has led to identification of four relevant CYPs, with the characterized function of CYP728B70 further providing insight into this previously enigmatic CYP family, as well as demonstrating the utility of this approach to increasing the yield of this promising pharmaceutical agent.

\section{Methods}

Genome sequencing and assembly. A T. wilfordii cultivar was used for sequencing (Supplementary Note 1). Genomic DNA was extracted from leaves of T. wilfordii using the DNAsecure Plant Kit (TIANGEN) and broken into random fragments. Short-reads libraries were constructed according to the manufacturer's instructions (Illumina, San Diego, CA) and then sequenced on Illumina Hiseq Xten. For long-read DNA sequencing, $60 \mathrm{~kb}$ Single Molecule Real Time (SMRT) long-read library were sequenced on the PacBio Sequel platform (75.79 Gb data, 207-fold coverage of the genome). For 10X Genomics sequencing, a total of 119.75 $\mathrm{Gb}$ (327-fold coverage of the genome) data were sequenced on the Illumina Hiseq $\mathrm{X}$-Ten (Supplementary Table 2).

De novo assembly of the long reads from the PacBio SMRT Sequencer was performed using FALCON (https://github.com/PacificBiosciences/FALCON/) ${ }^{49}$ To obtain enough corrected reads, the longest coverage of subreads were firstly selected as seed reads to correct sequence errors. Then, error-corrected reads were aligned to each other and assembled into genomic contigs using FALCON with the following parameters: length_cutoff_pr $=10,000, \max \_$diff $=95$, and max_cov $=$ 105. Then, genomic contigs were polished using Quiver ${ }^{50}$, which yielded an assembly with a contig N50 size of $4.36 \mathrm{Mb}$. The total length of this assembly version was $348.38 \mathrm{Mb}$. Then, we used BWA-MEM to align the 10X Genomics data to the assembly using default settings ${ }^{51}$. Scaffolding was performed by FragScaff with the barcoded sequencing reads ${ }^{52}$. Last, Pilon ${ }^{53}$ was used to perform error correction based on the Illumina sequences, generating a genome with a scaffold $\mathrm{N} 50$ size of $6.48 \mathrm{Mb}$. The total length of this assembly version was $349.91 \mathrm{Mb}$. Subsequently, the Hi-C sequencing data were aligned to the assembled scaffolds by BWA-mem ${ }^{50}$ and the scaffolds were clustered onto chromosomes with LACHESIS (http://shendurelab.github.io/LACHESIS/), the final genome was $348.53 \mathrm{Mb}$ and the contig and scaffold $\mathrm{N} 50$ were $4.36 \mathrm{Mb}$ and $13.52 \mathrm{Mb}$, respectively

(Supplementary Tables 3-7 and Supplementary Note 1). 
a

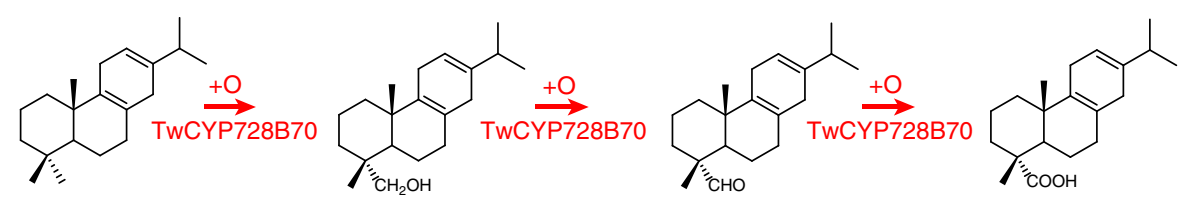<smiles>CC(C)c1ccc2c(c1)CCC1C2CCCC1(C)C</smiles>

8,12-Abietadienol (4)

8,12-Abietadienal

8,12-Abietadienoic acid (2) Spontaneous

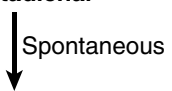
$\downarrow$ Spontaneous<smiles>CC(C)c1ccc2c(c1)CCC1C(=C2)CC2C1(C)CCC[C@]2(C)O</smiles>

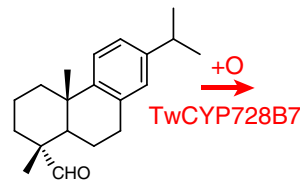

Abietatriene

Dehydroabietinol (3)

Dehydroabietinal

Dehydroabietic acid (1)

Triptolide

b

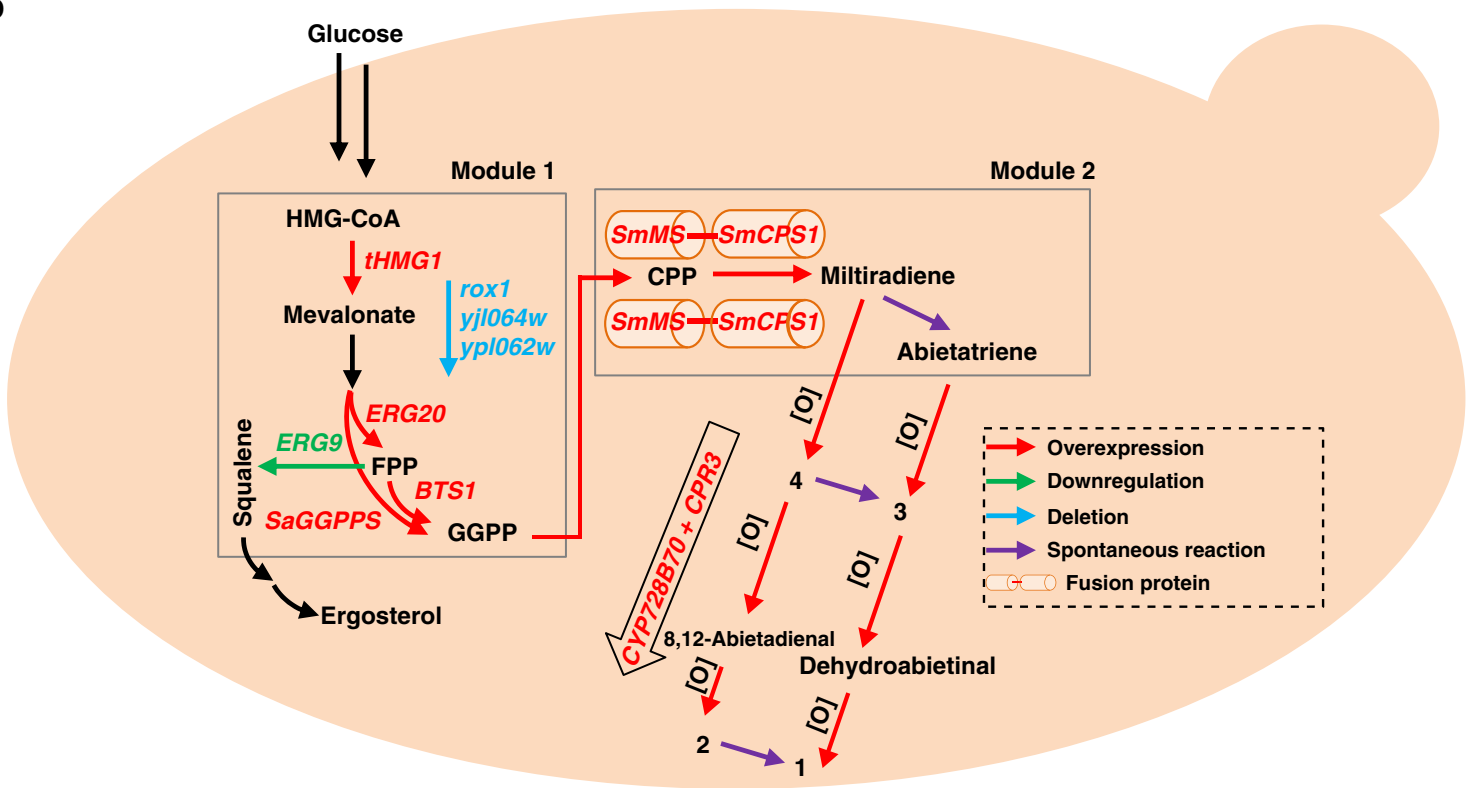

Fig. 4 Analysis of triptolide pathway and metabolic engineering. a Three reactions presumably catalyzed by CYP728B70 to convert miltiradiene to dehydroabietic acid. b Establishing the metabolic pathways in yeast for production of diterpene alcohols and acids. In module 1, rox1, ypl062w, yjl06w4 were knocked out, and ERG9 was down-regulated, and tHMG1, ERG20, BTS1, SaGGPPS were overexpressed to improve the production of GGPP. In module 2, double SmMS-SmCPS1 fusion modules were introduced into the yeast chromosome for the production of miltiradiene. Co-expression of TwCYP728B70 and TwCPR3 leads to the observed production of the derived alcohols and acids.

Genome annotation. A total of $52.36 \%$ repeat sequences in the genome were annotated. Among them, TEs were searched by combining de novo-based and homology-based approaches using RepeatModeler (http://www.repeatmasker.org/ RepeatModeler/), LTR_FINDER (http://tlife.fudan.edu.cn/ltr_finder/), RepeatScout (http://www.repeatmasker.org/), RepeatMasker (version 3.3.0) (http://www. repeatmasker.org/), and RepeatProteinMask (http://www.repeatmasker.org/). Tandem repeats were detected using Tandem Repeats Finder (TRF) ${ }^{54}$ (Supplementary Fig. 2 and Supplementary Table 10). Gene structures were predicted with a combination of homology-based prediction, de novo prediction and transcriptome-based prediction in the genome (Supplementary Fig. 3, Supplementary Tables 8 and 9). We then generated functional assignments of the T. wilfordii genes with BLAST in public protein databases, including SwissProt (https://web.expasy.org/docs/swiss-prot_guideline.html), NR, InterPro (V32.0) ${ }^{55}$, Pfam (V27.0) $)^{56}$ and KEGG (https://www.kegg.jp/). Finally, 99.6\% of all genes in the genome were predicted to be functional (Supplementary Table 11). Noncoding RNA was predicted using de novo and homology search methods (Supplementary Table 12 and Supplementary Note 2).

Genome evolution. We conducted expansion and contraction analysis using the CAFÉ program ${ }^{57}$ (Supplementary Table 15) and identified the positively selected genes in T. wilfordii using MUSCLE ${ }^{58}$ (Supplementary Tables 16 and 17). To identify the WGD events in the T. wilfordii genome, we used McscanX ${ }^{59}$ to determine syntenic blocks (regions with at least five genes) and calculated 4DTv for all gene pairs found in each syntenic segment. The synonymous substitution rate $(K s)$ values of $T$. wilfordii syntenic block genes were calculated with the codeml program of the PAML ${ }^{60}$ package. We performed synteny analysis on T. wilfordii and $V$. vinifera to confirm that $T$. wilfordii had undergone another WGT event.

Evolution of triptolide biosynthesis genes. To investigate the genes involved in triptolide biosynthesis, we first retrieved protein sequences from Arabidopsis thaliana genes, including ACAT, CMK, DXR, DXS, FPS, GGPPS, GPS, HDR, HDS, HMGR, HMGS, IDI, MCT, MCS, MVK, MVD, and PMK from the NCBI database The CPS and MS protein sequences in T. wilfordii were previously cloned ${ }^{28,31}$. Then, using these homologs as queries, we identified the candidates in T. wilfordii and 13 other plant species, including Carica papaya, Daucus carota, Dimocarpus longan, Glycine max, Gossypium raimondii, Glycyrrhiza uralensis, Oryza sativa, Prunus persica, Populus trichocarpa, Solanum lycopersicum, Salvia miltiorrhiza, Theobroma cacao, Vitis vinifera using BLASTP with an E-value cutoff of $1 \mathrm{e}^{-5}$. The aligned hits with at least $50 \%$ coverage of seed protein sequences and $>50 \%$ protein sequence identity were selected as homologs. Then, the domains of these homologs 
were predicted by PFAM (http://pfam.xfam.org/). Only genes that had the same protein domain were considered to be homologs. The TPS and CYP450 genes were predicted using $\mathrm{hmmsearch}{ }^{61}$ in conjunction with the TPS hmm model (PF01397 and PF03936) and CYP450 hmm model (PF00067) from Pfam, respectively. We constructed phylogenetic trees of each identified triptolide biosynthetic gene family from T. wilfordii and Arabidopsis thaliana. Amino acid sequence alignments of the identified triptolide biosynthetic genes from T. wilfordii and Arabidopsis thaliana were performed using MUSCLE ${ }^{58}$, and the alignment data were used to construct phylogenetic trees using RAxML ${ }^{62}$ with the maximum likelihood method. The timing of the divergence of duplicates in each triptolide biosynthesis gene was estimated based on the calculation of the synonymous substitution rate (Ks) using the codeml program of the PAML ${ }^{60}$ package. The calculated $K s$ value was then converted to the divergence time according to $T=K s / 2 \mathrm{r}$, where $\mathrm{r}$ represents a substitution rate of $6.5 \times 10^{-9}$ mutations per site per year for eudicots (Supplementary Table 19).These gene pairs were not adjacent in the distribution of the genome (Supplementary Data 2).

\section{Suspension cell elicitation and sample preparation. It has previously been} shown that the application of the plant defence signaling molecule MeJA could increase terpenoid production and affect the transcript levels of the genes involved in the related pathways ${ }^{28,63}$. Cell samples were harvested at $0,4,8,12,24,36,48$, $72,96,120,240,360$, and $480 \mathrm{~h}$ after the addition of MeJA and DMSO. Solid samples were homogenized by Mixer Mill MM 400 of Retsch (Retsch GmbH, 42781 Haan, Germany) and stored at $-80^{\circ} \mathrm{C}$ for at least $4 \mathrm{~h}$ prior to freeze drying for $48 \mathrm{~h}$ (Alpha 1-2 LDplus, Germany). For each sample, $60 \mathrm{mg}$ aliquots were soaked in $1.5 \mathrm{~mL}$ of $80 \%(\mathrm{v} / \mathrm{v})$ methanol overnight at room temperature and then dissolved in an ultrasonic water bath for $60 \mathrm{~min}$. The supernatant was filtered through a $0.22-\mu \mathrm{m}$ membrane filter (FitMax Syringe Filter, $13 \mathrm{~mm} 0.22 \mu \mathrm{m}$ ) for further analysis.

UPLC/Q-TOF MS analysis. All analyses were performed on a UPLC/Q-TOF MS system (Waters Corp., Milford, MA, USA). The UPLC separation was performed using a Waters ACQUITY UPLC HSS T3 analytical column $(2.1 \mathrm{~mm} \times 100 \mathrm{~mm}$, $1.8 \mu \mathrm{m})$ kept at $40^{\circ} \mathrm{C}$ and a Waters Acquity UPLC ${ }^{\mathrm{TM}}$ I-Class system. The mobile phase was pumped with a mixture of $0.1 \%(\mathrm{v} / \mathrm{v})$ aqueous acetic acid (A) and acetonitrile (B) at a flow rate of $0.5 \mathrm{~mL} \mathrm{~min}{ }^{-1}$. Gradient elution was performed as follows: $0 \mathrm{~min}$ at $30 \% \mathrm{~B}, 6 \mathrm{~min}$ at $45 \% \mathrm{~B}, 18 \mathrm{~min}$ at $60 \% \mathrm{~B}$, and $23 \mathrm{~min}$ at $90 \% \mathrm{~B}$. The TOF MS experiments were performed on the Xevo G2-S QTOF MS system. The experiment was performed in ESI (+) ionization modes, and the data acquisition modes were MSE continuum. The capillary voltage was $0.5 \mathrm{KV}$, and the cone voltage was $40 \mathrm{~V}$. The source and desolvation temperatures were $100^{\circ} \mathrm{C}$ and $450{ }^{\circ} \mathrm{C}$, respectively. The desolvation gas flow was $900 \mathrm{~L} \mathrm{~h}^{-1}$. The ramp collision energy was set as $20-40 \mathrm{eV}$ for the high-energy scans. The MS range of data acquisition was 50-1500 Da. A lock spray with leucine enkephalin $\left(200 \mathrm{pg} \mu \mathrm{L}^{-1}\right.$, $10 \mu \mathrm{L} \mathrm{min}^{-1}$ ) was used as the reference (m/z 556.2771 ESI (+)) to maintain the mass accuracy.

Data analysis of metabolic and transcriptional profiles. All mass spectral data were imported into Progenesis QI for data processing, then were grouped and exported to Ezinfo for principal component analysis (PCA $)^{64}$. PCA is a very useful statistical method for defining the dimensions of large data sets and identifying significant signals ${ }^{65}$. The data set was log normalized, and PCA and OPLS-DA analyses were performed to determine overall differences in metabolites. An ANOVA with a significance level of $P<0.01$ and max-fold $>2$ was subsequently performed on the doubly filtered peaks to identify metabolites that did not change significantly in response to MeJA treatment. Furthermore, peaks with no fragments were removed.

We selected metabolite peaks and genes including all CYP, CPS1 and MS for network regulation analysis. Both data sets of accumulation of metabolic and gene expression were normalized separately, and correlation network analysis was used to establish gene-to-metabolite coregulation patterns ${ }^{41}$. The Pearson correlation coefficient was calculated by the PCC method in the R platform between each set of variables (either metabolite or gene) across the profiles, and significant positive correlations with $p$-value $<0.05$ were detected between genes and metabolites. The correlation network was analyzed by the Cytoscape software (version 3.6.1 ${ }^{66}$.

\section{Construction of miltiradiene-producing yeast strain. To construct a} miltiradiene-producing strain, the corresponding biosynthetic pathway was introduced into yeast. First, the CRISPR/Cas9 system was applied to improve the MVA pathway flux by knocking out three transcriptional regulators (roxl, ypl062 $\mathrm{w}$ and yjl06w4) and knocking down erg9 in the yeast BY-T20 strain (BY4742, $\Delta \operatorname{Trp} 1$, Trp1::His3-P $P_{P G K 1}-B T S 1 / E R G 20-T_{A D H 1}-P_{T D H 3}-S a G G P P S-T_{T P I 1}-P_{T E F 1}-t H M G 1-$ $T_{C Y C 1}$ ), generating a GGPP-producing yeast strain named BY-HZ16 ${ }^{44,67-69}$.

The fusion of SmCPS1 and SmMS from Salvia miltiorrhiza was reported to possess great potential to synthesize miltiradiene via GGPP ${ }^{70}$. The single or double SmMS-SmCPS1 fusion module was cloned and integrated into the yeast chromosome by the M2S integration method ${ }^{71}$. Briefly, SmMS-SmCPS1 was amplified with the addition of a BsaI digestion site and ligated with head-to-head promoters ( $p T D H 3-p A D H 1)$ into the bi-terminator vector T1-(tTPI1-tPGI1), resulting in the plasmids T1-(SmMS-SmCPS1) and T1-(SmMS-SmCPS1)-(SmMSSmCPS1). Two terminators were inserted into the scaffold plasmid, with dedicated homologous arms (L1 and L2) lying on both sides. The integration site YPRCA15 was chosen as the target locus, and Ura3 was chosen as the selection marker. Each expression cassette with designed to have homologous arms (primers: L1-F/L2-R) was amplified individually. The selection marker module and integration homologous arm module (15Site1-Ura3-L1 and L2-15site2) were also amplified. All the amplified fragments were used to co-transform BY-HZ16 for assembly and integration, and transformants were selected on synthetic dropin medium-Ura-His (SD-Ura-His) containing $20 \mathrm{~g} \mathrm{~L}^{-1}$ glucose and $18 \mathrm{~g} \mathrm{~L}^{-1}$ agar. Positive transformants were verified by sequencing, yielding the strains PS1 and PS2 (Supplementary Fig. 23). All strains are listed in Supplementary Table 21. Al primers used in vector construction are listed in Supplementary Data 7.

CYP screening based on the miltiradiene-producing strain. The gene-tometabolite network analysis and specific-gene analysis in T. wilfordii were performed, and 10 highly expressed CYP genes were chosen as candidates for investigation. The RNAi results showed that 4 CYPs were most likely to regulate triptolide biosynthesis and were then selected to react with abietane-type diene olefin miltiradiene. First, to simplify the fermentation procedure, the constitutive promoters pPGK1-pTEF2 were cloned into the BamHI and NotI sites of the pESCLeu vector to replace the inducible promoters $p G A L 1-p G A L 10$, yielding the plasmid Leu-PT. Second, cytochrome P450 reductase 3 (CPR3) was inserted into the NotI site of the plasmid Leu-PT according to the pEASY-Uni Seamless Cloning and Assembly Kit (TransGen Biotech, Beijing, China), resulting in the plasmid Leu-PTCPR3. Then, each CYP gene was introduced into the BamHI site of the plasmid Leu-PT-CPR3. All the resulting CYP expression plasmids were individually introduced into strain PS2 following the user manual of the Frozen-EZ Yeast Transformation II KitTM (Zymo Research, USA) for product identification. All primers used for CYP gene screening are listed in Supplementary Data 7.

Three colonies were picked for each genotype and used to inoculate $5 \mathrm{~mL}$ of synthetic dropin medium -Ura-His-Leu (SD-Ura-His-Leu) containing $20 \mathrm{~g} \mathrm{~L}^{-1}$ glucose. The cells were grown in a shaker at $30^{\circ} \mathrm{C}$ and $230 \mathrm{rpm}$ for $48 \mathrm{~h}$, after which the resulting seed cultures were transferred into fresh medium at a ratio of 1:50 and fermented under the same conditions for 3 days. Yeast cells were lysed using a nano homogenizer (AH-1500, ATS Engineering Limited, Canada) and then extracted twice with an equal volume of ethyl acetate. Anhydrous sodium sulfate was added to remove residual water, and the combined organic phases were dried and methylated with (trimethylsilyl)diazomethane (Aladdin Industrial Inc.,

Shanghai, China) ${ }^{43,72}$. The methylated samples were re-dried and then dissolved in $100 \mu \mathrm{L}$ of ethyl acetate for gas chromatography-mass spectrometry (GC-MS) using a Thermo TRACE 1310/TSQ 8000 gas chromatograph equipped with a TG-5 MS $(30 \mathrm{~m} \times 0.25 \mathrm{~mm} \times 0.25 \mu \mathrm{m})$ capillary column. The GC conditions were as follows: the sample $(1 \mu \mathrm{L})$ was injected in split mode $(20: 1)$ at $250^{\circ} \mathrm{C}$ under a He flow rate of $1 \mathrm{~mL} \mathrm{~min}^{-1}$, the GC oven temperature was programmed to rise from an initial $40^{\circ} \mathrm{C}$ at $20^{\circ} \mathrm{C} \mathrm{min}^{-1}$ to $200^{\circ} \mathrm{C}$ and at $15^{\circ} \mathrm{C} \mathrm{min}-1$ to $250{ }^{\circ} \mathrm{C}$, then to $270{ }^{\circ} \mathrm{C}$ at $1.5^{\circ} \mathrm{C} \mathrm{min}{ }^{-1}$. The ion trap heating temperature was $250^{\circ} \mathrm{C}$. The electron energy was $70 \mathrm{eV}$. Spectra were recorded in the range of $40-500 \mathrm{~m} / \mathrm{z}$.

\section{In vivo assays for TwCYP728B70 activity. The pESC-Leu::(CPR3 +}

TwCYP728B70) construct was transformed into the yeast strain WAT11, which enables the catalytic activity of plant CYPs by expressing an Arabidopsis CPR ${ }^{45}$. Transformants were selected on synthetic dropin medium SD-Leu plates containing $20 \mathrm{~g} \mathrm{~L}^{-1}$ glucose. The cells were grown in a shaker at $30^{\circ} \mathrm{C}$ and $230 \mathrm{rpm}$ for $48 \mathrm{~h}$, then transferred into $50 \mathrm{~mL}$ of fresh medium at a ratio of 1:50 and fermented under the same conditions for $12 \mathrm{~h}$. To confirm engineered yeast strains for oxidative transformation of diterpenoid substrates, miltiradiene, abietatriene, 8,12 abietadienol, or dehydroabietinol (in methanol) was added to the yeast cultures to final concentrations of $100 \mu \mathrm{M}$ and fermented for another $48 \mathrm{~h}$. Yeast cells were lysed, extracted twice with an equal volume of ethyl acetate, and methylated before GC-MS analysis, as described above.

RNAi of the candidate CYP genes in T. wilfordii. The fragments of these 10 candidate CYP genes were amplified using Phusion ${ }^{\circledR}$ High-Fidelity DNA Polymerase (New England Biolabs, USA) and inserted into the RNAi vector pK7GWIWG2D according to the Gateway procedure (Invitrogen, USA), and the resulting vectors were verified by complete sequencing.

Suspension cells in the logarithmic growth phase were chose and precultured on Murashige and Skoog (MS) solid medium containing $0.5 \mathrm{mg} \mathrm{L}^{-1} 2,4-\mathrm{D}, 0.1 \mathrm{mg} \mathrm{L}^{-1}$ $\mathrm{KT}, 0.5 \mathrm{mg} \mathrm{L}^{-1} \mathrm{IBA}$ and $30 \mathrm{~g} \mathrm{~L}^{-1}$ sucrose $(\mathrm{pH}=5.8)$ for 7 days. Then, the recombinant plasmid DNA mixed with Au microparticles were transformed into the suspension cells through bombardment using a biolistic gene gun (PDS 1000/ $\mathrm{He}$, Bio-Rad). Each transformation was carried out two times. The bombarded suspension cells were cultured for another 7 days before harvesting for qRT-PCR and UPLC analysis ${ }^{73}$ 
Overexpression of TwCYP728B70. Vector pH7WG2D (Invitrogen) was used to overexpress $T w C Y P 728 B 70$ in suspension cells following the protocol mentioned above. The resulting recombinant cells were harvested for qRT-PCR and UPLC analysis after being cultured for $3,5,7$, and 9 days $^{73}$.

Heterologous expression of CYP in yeast and in vitro assays. Each CYP gene was inserted into the BamHI site of the pESC-Leu vector according to the pEASYUni Seamless Cloning and Assembly Kit. The pESC-Leu::CYP construct was verified by complete gene sequencing, which was transformed into the yeast strain WAT11. The cells were grown first in $100 \mathrm{~mL}$ of SD-Leu liquid medium with $20 \mathrm{~g} \mathrm{~L}^{-1}$ glucose in a shaker at $30^{\circ} \mathrm{C}$ and $230 \mathrm{rpm}$ to an $\mathrm{OD}_{600}$ of 2-3. Cells were centrifuged and resuspended in $200 \mathrm{~mL}$ of yeast peptone galactose (YPL) induction medium ( $10 \mathrm{~g} \mathrm{~L}^{-1}$ yeast extract, $20 \mathrm{~g} \mathrm{~L}^{-1}$ bactopeptone, and $20 \mathrm{~g} \mathrm{~L}^{-1}$ galactose) and grown for $12 \mathrm{~h}$ at $30^{\circ} \mathrm{C}$ to induce recombinant protein expression. Microsomes were prepared based on the reported method with some modifications ${ }^{74,75}$. Briefly, the induced cells were centrifuged $\left(1914 \times g, 4{ }^{\circ} \mathrm{C}, 5 \mathrm{~min}\right)$ and resuspended in $20 \mathrm{~mL}$ of TEK buffer (50 mM Tris-HCl, pH 7.4, $1 \mathrm{mM} \mathrm{EDTA,} 0.1 \mathrm{M} \mathrm{KCl})$, then left at room temperature for $5 \mathrm{~min}$. Cells were centrifuged again $\left(1,914 \times g, 4^{\circ} \mathrm{C}, 5 \mathrm{~min}\right)$ and resuspended in $50 \mathrm{~mL}$ of TESB buffer $(50 \mathrm{mM}$ Tris- $\mathrm{HCl}$, $\mathrm{pH} 7.4,1 \mathrm{mM}$ EDTA, 0.6 $\mathrm{M}$ sorbitol), then left on ice for $10 \mathrm{~min}$. The cell suspension was lysed for $7 \mathrm{~min}$ at 4 ${ }^{\circ} \mathrm{C}$ and 12,000 psi using a nano homogenize machine (AH-1500, ATS Engineering Limited, Canada), then centrifuged $\left(17,266 \times g, 4^{\circ} \mathrm{C}, 15 \mathrm{~min}\right)$ to collect the supernatant. $\mathrm{NaCl}$ (final concentration of $0.15 \mathrm{M}$ ) and polyethylene glycol (PEG) -4000 (final concentration of $0.1 \mathrm{~g} \mathrm{~mL}^{-1}$ ) were added to the supernatant, and left on ice for $15 \mathrm{~min}$. The microsomal fractions were collected by centrifugation $(17,266 \times g, 4$ $\left.{ }^{\circ} \mathrm{C}, 15 \mathrm{~min}\right)$, and resuspended in TEG buffer $(50 \mathrm{mM}$ Tris- $\mathrm{HCl}, \mathrm{pH} 7.4,1 \mathrm{mM}$ EDTA, $20 \%(\mathrm{v} / \mathrm{v})$ glycerol), which can be kept frozen at $-80^{\circ} \mathrm{C}$ for months.

In vitro enzymatic activity assays were carried out on a shaking incubator (150 $\mathrm{rpm}$ ) at $30^{\circ} \mathrm{C}$ for $4 \mathrm{~h}$ in $500 \mu \mathrm{L}$ of $100 \mathrm{mM}$ Tris- $\mathrm{HCl}, \mathrm{pH} 7.5$, containing $0.5 \mathrm{mg}$ of total microsomal proteins and $500 \mu \mathrm{M} \mathrm{NADPH}$, along with a regenerating system (consisting of $5 \mu \mathrm{M}$ FAD, $5 \mu \mathrm{M}$ FMN, $5 \mathrm{mM}$ glucose-6-phosphate, 1 unit mL $\mathrm{mL}^{-1}$ glucose-6-phosphate dehydrogenase), and $100 \mu \mathrm{M}$ substrates. Reactions were stopped by the addition of $500 \mu \mathrm{L}$ of methanol and used for UPLC analysis. Negative control reactions were carried out with microsomal preparations from recombinant yeast transformed with empty pESC-Leu.

Fermentation. To engineer yeast for the production of intermediates involved in triptolide biosynthesis and obtain enough compound for structural characterization, the strain PS2 containing the plasmid Leu-PT-CPR3::TwCYP728B70 was used to inoculate $50 \mathrm{~mL}$ of SD-Ura-His-Leu medium in a $250 \mathrm{~mL}$ shake flask at $30^{\circ} \mathrm{C}$ and $230 \mathrm{rpm}$ for $24 \mathrm{~h}$. The entire culture volume was transferred into $500 \mathrm{~mL}$ of fresh seed medium and incubated for $24 \mathrm{~h}$, then transferred into $2 \mathrm{~mL}$ of fresh seed medium and incubated for another $24 \mathrm{~h}$. The seed medium was then used to inoculate $8 \mathrm{~L}$ of fermentation medium in a New Brunswick BioFlo/CelliGen 115 bioreactor (Eppendorf, Germany) with a maximal working volume of $14 \mathrm{~L}$.

The fermentation was performed at $30^{\circ} \mathrm{C}$. During fermentation, the $\mathrm{pH}$ was maintained at 5.0 with the automatic addition of ammonium hydroxide, the agitation rate was kept between 200 and $600 \mathrm{rpm}$, and the dissolved oxygen was kept above $40 \%$. Concentrated glucose solution $(40 \%$, wt $/ \mathrm{vol})$ was fed periodically to keep the glucose concentration above $1.0 \mathrm{~g} \mathrm{~L}^{-1}$. Additionally SD-Ura-His-Leu medium was fed after the initial $30 \mathrm{~h}$ of fermentation. The culture was then harvested by extraction after $90 \mathrm{~h}$ of total fermentation time.

Yeast cells were concentrated to $1 \mathrm{~L}$, then lysed using the nano homogenizer and then extracted ten times with an equal volume of ethyl acetate. Anhydrous sodium sulfate was added to remove residual water, and the organic fractions were pooled and dried using a Nitrogen Evaporator (Baojingkeji, Henan, China). The yellow oily liquid $(15.5 \mathrm{~g})$ was chromatographed on LiChroprep ${ }^{\circledast}$ Si60 $(40-63 \mu \mathrm{m}$, Merck, MA, USA) with a stepwise gradient of petroleum ether-hexane (v/v, 10:1) to obtain the fraction $(\sim 500 \mathrm{~mL})$. The fraction was re-dried and then dissolved in 500 $\mu \mathrm{L}$ of acetonitrile. Preparative HPLC was performed on Agilent 1260 Infinity HighPerformance Liquid Chromatography System with a Shim-pack GIST C18 (250 × $4.6 \mathrm{mml} . \mathrm{D}$., $5 \mu \mathrm{m}$, SHIMADZU, Kyoto, Japan). The mobile phase, consisting of a mixture of water (A) and acetonitrile (B), was pumped at a flow rate of $1 \mathrm{ml} \mathrm{min}^{-1}$ and the eluate was monitored at $200 \mathrm{~nm}$. The gradient elution was programmed as follows: $0 \mathrm{~min}$ at $65 \% \mathrm{~B}, 50 \mathrm{~min}$ at $65 \% \mathrm{~B}$. The injection volume was $20 \mu \mathrm{L}$.

Reporting summary. Further information on research design is available in the Nature Research Reporting Summary linked to this article.

\section{Data availability}

The data supporting the findings of this work are available within the paper and its Supplementary Information files. A reporting summary for this Article is available as a Supplementary Information file. The data sets generated and analyzed during this study are available from the corresponding author upon request. The genome sequence data and transcriptome sequence data for $T$. wilfordii have been deposited under NCBI BioProject number PRJNA542587 and the final assembly is available at GenBank under the accession number JAAARO000000000. The source data underlying Figs. 1, 2, 3a, 3b, and $3 \mathrm{f}$ are provided as a Source Data file.
Received: 28 April 2019; Accepted: 1 February 2020;

Published online: 20 February 2020

\section{References}

1. Liu, Y. et al. Extracts of Tripterygium wilfordii Hook F in the treatment of rheumatoid arthritis: a systemic review and meta-analysis of randomised controlled trials. Evid. Based Complement Altern. Med. 2013, 410793 (2013).

2. Corson, T. W. \& Crews, C. M. Molecular understanding and modern application of traditional medicines: triumphs and trials. Cell 130, 769-774 (2007).

3. Chen, Y. W. et al. Triptolide exerts anti-tumor effect on oral cancer and $\mathrm{KB}$ cells in vitro and in vivo. Oral. Oncol. 45, 562-568 (2009).

4. Titov, D. V. et al. XPB, a subunit of TFIIH, is a target of the natural product triptolide. Nat. Chem. Biol. 7, 182-188 (2011).

5. Manzo, S. G. et al. Natural product triptolide mediates cancer cell death by triggering CDK7-dependent degradation of RNA polymerase II. Cancer Res. 72, 5363-5373 (2012).

6. Chugh, R. et al. A preclinical evaluation of Minnelide as a therapeutic agent against pancreatic cancer. Sci. Transl. Med. 4, 156ra139 (2012).

7. Zheng, Y., Zhang, W. J. \& Wang, X. M. Triptolide with potential medicinal value for diseases of the central nervous system. CNS Neurosci. Ther. 19, 76-82 (2013).

8. Mujumdar, N. et al. Triptolide induces cell death in pancreatic cancer cells by apoptotic and autophagic pathways. Gastroenterology 139, 598-608 (2010).

9. Wang, L., Xu, Y., Fu, L., Li, Y. \& Lou, L. (5R)-5-hydroxytriptolide (LLDT-8), a novel immunosuppressant in clinical trials, exhibits potent antitumor activity via transcription inhibition. Cancer Lett. 324, 75-82 (2012).

10. Rivard, C. et al. Inhibition of epithelial ovarian cancer by Minnelide, a watersoluble pro-drug. Gynecol. Oncol. 135, 318-324 (2014).

11. Arora, N. et al. Tu1964 Minnelide: a novel therapeutic agent for gastric adenocarcinoma. Gastroenterology 148, S-947 (2015).

12. Zeng, F. et al. Simultaneous quantification of 18 bioactive constituents in Tripterygium wilfordii using liquid chromatography-electrospray ionizationmass spectrometry. Planta Med. 79, 797-805 (2013).

13. Zhou, Z. L., Yang, Y. X., Ding, J., Li, Y. C. \& Miao, Z. H. Triptolide: structural modifications, structure-activity relationships, bioactivities, clinical development and mechanisms. Nat. Prod. Rep. 29, 457-475 (2012).

14. Buckanin, R. S., Chen, S. J., Frieze, D. M., Sher, F. T. \& Berchtold, G. A. Total synthesis of triptolide and triptonide. J. Am. Chem. Soc. 102, 1200-1201 (1980).

15. Lai, C. K. et al. Total synthesis of racemic triptolide and triptonide. J. Org Chem. 47, 2364-2369 (1982).

16. Van Tamelen, E. E., Demers, J. P., Taylor, E. G. \& Koller, K. Total synthesis of l-triptonide and l-triptolide. J. Am. Chem. Soc. 102, 5424-5425 (1980).

17. Goncalves, S., Hellier, P., Nicolas, M., Wagner, A. \& Baati, R. Diastereoselective formal total synthesis of $(+/-)$-triptolide via a novel cationic cyclization of 2-alkenyl-1,3-dithiolane. Chem. Commun. (Camb.) 46, 5778-5780 (2010).

18. Kutney, J. P. et al. Cyto-toxic diterpenes triptolide, tripdiolide, and cyto-toxic triterpenes from tissue-cultures of Tripterygium wilfordii. Can. J. Chem. 59, 2677-2683 (1981).

19. Miao, G. P. et al. Elicitation and in situ adsorption enhanced secondary metabolites production of Tripterygium wilfordii Hook. f. adventitious root fragment liquid cultures in shake flask and a modified bubble column bioreactor. Bioprocess Biosyst. Eng. 37, 641-650 (2014).

20. Su, P. et al. Characterization of eight terpenoids from tissue cultures of the Chinese herbal plant, Tripterygium wilfordii, by high-performance liquid chromatography coupled with electrospray ionization tandem mass spectrometry. Biomed. Chromatogr. 28, 1183-1192 (2014).

21. Inabuy, F. S. et al. Biosynthesis of diterpenoids in Tripterygium adventitious root cultures. Plant Physiol. 175, 92-103 (2017).

22. Miao, G. P. et al. Aggregate cell suspension cultures of Tripterygium wilfordii Hook. f. for triptolide, wilforgine, and wilforine production. Plant Cell Tissue Organ Cult. 112, 109-116 (2013).

23. Martin, V. J., Pitera, D. J., Withers, S. T., Newman, J. D. \& Keasling, J. D. Engineering a mevalonate pathway in Escherichia coli for production of terpenoids. Nat. Biotechnol. 21, 796-802 (2003).

24. Ro, D. K. et al. Production of the antimalarial drug precursor artemisinic acid in engineered yeast. Nature 440, 940-943 (2006).

25. Paddon, C. J. et al. High-level semi-synthetic production of the potent antimalarial artemisinin. Nature 496, 528-532 (2013).

26. Farhi, M. et al. Generation of the potent anti-malarial drug artemisinin in tobacco. Nat. Biotechnol. 29, 1072-1074 (2011).

27. Kutney, J. P. \& Han, K. Studies with plant-cell cultures of the Chinese herba plant, Tripterygium wilfordii. Isolation and characterization of diterpenes. Recl. Trav. Chim. Pays-Bas 115, 77-93 (1996). 
28. $\mathrm{Su}, \mathrm{P}$. et al. Identification and functional characterization of diterpene synthases for triptolide biosynthesis from Tripterygium wilfordii. Plant J. 93, 50-65 (2018)

29. Gao, W. et al. A functional genomics approach to tanshinone biosynthesis provides stereochemical insights. Org. Lett. 11, 5170-5173 (2009).

30. Zi, J. \& Peters, R. J. Characterization of CYP76AH4 clarifies phenolic diterpenoid biosynthesis in the Lamiaceae. Org. Biomol. Chem. 11, 7650-7652 (2013).

31. Hansen, N. L. et al. The terpene synthase gene family in Tripterygium wilfordii harbors a labdane-type diterpene synthase among the monoterpene synthase TPS-b subfamily. Plant J. 89, 429-441 (2017).

32. Nelson, D. \& Werck-Reichhart, D. A P450-centric view of plant evolution. Plant J. 66, 194-211 (2011).

33. Shen, Q. et al. The Genome of Artemisia annua provides insight into the evolution of Asteraceae family and artemisinin Biosynthesis. Mol. Plant 11, 776-788 (2018).

34. Liu, X. et al. The genome of medicinal plant Macleaya cordata provides new insights into benzylisoquinoline alkaloids metabolism. Mol. Plant 10, 975-989 (2017).

35. Parra, G., Bradnam, K. \& Korf, I. CEGMA: a pipeline to accurately annotate core genes in eukaryotic genomes. Bioinformatics 23, 1061-1067 (2007).

36. Simao, F. A., Waterhouse, R. M., Ioannidis, P., Kriventseva, E. V. \& Zdobnov, E. M. BUSCO: assessing genome assembly and annotation completeness with single-copy orthologs. Bioinformatics 31, 3210-3212 (2015).

37. Crow, K. D. \& Wagner, G. P. Investigators ST-NY. Proceedings of the SMBE Tri-National Young Investigators' Workshop 2005. What is the role of genome duplication in the evolution of complexity and diversity? Mol. Biol. Evol. 23, 887-892 (2006).

38. Nutzmann, H. W. \& Osbourn, A. Gene clustering in plant specialized metabolism. Curr. Opin. Biotechnol. 26, 91-99 (2014).

39. Guo, L. et al. The opium poppy genome and morphinan production. Science 362, 343-347 (2018)

40. Shang, Y. et al. Biosynthesis, regulation, and domestication of bitterness in cucumber. Science 346, 1084-1088 (2014).

41. Mounet, F. et al. Gene and metabolite regulatory network analysis of early developing fruit tissues highlights new candidate genes for the control of tomato fruit composition and development. Plant Physiol. 149, 1505-1528 (2009).

42. Liu, X. et al. Engineering yeast for the production of breviscapine by genomic analysis and synthetic biology approaches. Nat. Commun. 9, 448 (2018).

43. $\mathrm{Su}, \mathrm{P}$. et al. Probing the single key amino acid responsible for the novel catalytic function of ent-kaurene oxidase supported by NADPHcytochrome P450 reductases in Tripterygium wilfordii. Front Plant Sci. 8 , 1756 (2017).

44. Dai, Z., Liu, Y., Huang, L. \& Zhang, X. Production of miltiradiene by metabolically engineered Saccharomyces cerevisiae. Biotechnol. Bioeng. 109, 2845-2853 (2012).

45. Urban, P., Mignotte, C., Kazmaier, M., Delorme, F. \& Pompon, D. Cloning, yeast expression, and characterization of the coupling of two distantly related Arabidopsis thaliana NADPH-cytochrome P450 reductases with P450 CYP73A5. J. Biol. Chem. 272, 19176-19186 (1997).

46. Ro, D. K., Arimura, G., Lau, S. Y., Piers, E. \& Bohlmann, J. Loblolly pine abietadienol/abietadienal oxidase PtAO (CYP720B1) is a multifunctional, multisubstrate cytochrome P450 monooxygenase. Proc. Natl Acad. Sci. USA 102, 8060-8065 (2005)

47. Hamberger, B., Ohnishi, T., Hamberger, B., Seguin, A. \& Bohlmann, J. Evolution of diterpene metabolism: Sitka spruce CYP720B4 catalyzes multiple oxidations in resin acid biosynthesis of conifer defense against insects. Plant Physiol. 157, 1677-1695 (2011).

48. Goossens, A. It is easy to get huge candidate gene lists for plant metabolism now, but how to get beyond? Mol. Plant 8, 2-5 (2015).

49. Chin, C. S. et al. Phased diploid genome assembly with single-molecule realtime sequencing. Nat. Methods 13, 1050-1054 (2016).

50. Chin, C. S. et al. Nonhybrid, finished microbial genome assemblies from longread SMRT sequencing data. Nat. Methods 10, 563-569 (2013).

51. $\mathrm{Li}, \mathrm{H}$. Toward better understanding of artifacts in variant calling from highcoverage samples. Bioinformatics 30, 2843-2851 (2014).

52. Adey, A. et al. In vitro, long-range sequence information for de novo genome assembly via transposase contiguity. Genome Res. 24, 2041-2049 (2014).

53. Walker, B. J. et al. Pilon: an integrated tool for comprehensive microbial variant detection and genome assembly improvement. PLOS ONE 9, e112963 (2014).

54. Benson, G. Tandem repeats finder: a program to analyze DNA sequences. Nucleic Acids Res. 27, 573-580 (1999).

55. Hunter, S. et al. InterPro: the integrative protein signature database. Nucleic Acids Res. 37, D211-215 (2009).
56. Finn, R. D. et al. Pfam: the protein families database. Nucleic Acids Res. 42, D222-230 (2014)

57. Han, M. V., Thomas, G. W. C., Lugo-Martinez, J. \& Hahn, M. W. Estimating gene gain and loss rates in the presence of error in genome assembly and annotation using CAFE 3. Mol. Biol. Evol. 30, 1987-1997 (2013).

58. Edgar, R. C. MUSCLE: multiple sequence alignment with high accuracy and high throughput. Nucleic Acids Res. 32, 1792-1797 (2004).

59. Tang, H. B. et al. Perspective-synteny and collinearity in plant genomes. Science 320, 486-488 (2008).

60. Yang, Z. PAML 4: phylogenetic analysis by maximum likelihood. Mol. Biol. Evol. 24, 1586-1591 (2007)

61. Finn, R. D., Clements, J. \& Eddy, S. R. HMMER web server: interactive sequence similarity searching. Nucleic Acids Res. 39, W29-W37 (2011).

62. Stamatakis, A. RAxML version 8: a tool for phylogenetic analysis and postanalysis of large phylogenies. Bioinformatics 30, 1312-1313 (2014).

63. Rischer, H. et al. Gene-to-metabolite networks for terpenoid indole alkaloid biosynthesis in Catharanthus roseus cells. Proc. Natl Acad. Sci. USA 103, 5614-5619 (2006).

64. Ghosson, H., Schwarzenberg, A., Jamois, F. \& Yvin, J. C. Simultaneous untargeted and targeted metabolomics profiling of underivatized primary metabolites in sulfur-deficient barley by ultra-high performance liquid chromatography-quadrupole/time-of-flight mass spectrometry. Plant Methods 14, 62 (2018).

65. Fukusaki, E. \& Kobayashi, A. Plant metabolomics: potential for practical operation. J. Biosci. Bioeng. 100, 347-354 (2005).

66. Shannon, P. et al. Cytoscape: asoftware environment for integrated models of biomolecular interaction networks. Genome Res 13, 2498-2504 (2003).

67. Dai, Z. et al. Metabolic engineering of Saccharomyces cerevisiae for production of ginsenosides. Metab. Eng. 20, 146-156 (2013).

68. Ozaydin, B., Burd, H., Lee, T. S. \& Keasling, J. D. Carotenoid-based phenotypic screen of the yeast deletion collection reveals new genes with roles in isoprenoid production. Metab. Eng. 15, 174-183 (2013).

69. Jakociunas, T. et al. Multiplex metabolic pathway engineering using CRISPR/ Cas9 in Saccharomyces cerevisiae. Metab. Eng. 28, 213-222 (2015).

70. Zhou, Y. J. et al. Modular pathway engineering of diterpenoid synthases and the mevalonic acid pathway for miltiradiene production. J. Am. Chem. Soc. 134, 3234-3241 (2012).

71. Li, S., Ding, W., Zhang, X., Jiang, H. \& Bi, C. Development of a modularized two-step (M2S) chromosome integration technique for integration of multiple transcription units in Saccharomyces cerevisiae. Biotechnol. Biofuels 9, 232 (2016).

72. Su, P. et al. Functional characterization of ent-copalyl diphosphate synthase, kaurene synthase and kaurene oxidase in the Salvia miltiorrhiza gibberellin biosynthetic pathway. Sci. Rep. 6, 23057 (2016).

73. Zhao, Y. et al. Genetic transformation system for woody plant Tripterygium wilfordii and its application to product natural celastrol. Front Plant Sci. 8, 2221 (2017)

74. Pompon, D., Louerat, B., Bronine, A. \& Urban, P. Yeast expression of animal and plant P450s in optimized redox environments. Methods Enzymol. 272, 51-64 (1996).

75. Guo, J. et al. CYP76AH1 catalyzes turnover of miltiradiene in tanshinones biosynthesis and enables heterologous production of ferruginol in yeasts. Proc Natl Acad. Sci. USA 110, 12108-12113 (2013).

\section{Acknowledgements}

The authors thank Y.T. and Z.L. (Datian Taoyuan State Forest Farm in Fujian Province, Datian, China) for providing plant material of T. wilfordii. We acknowledge support from the National Natural Science Foundation of China (No. 81773830, No. 81973418, No. 81891010, No. 81891013), the High-level Teachers in Beijing Municipal Universities in the Period of 13th Five-year Plan (No. CIT\&TCD20170324), the National Program for Special Support of Eminent Professionals, the Key Project at central government level: the ability establishment of sustainable use for valuable Chinese medicine resources (No. 2060302-1806-03), and the National Institutes of Health (GM109773 to R.J.P.).

\section{Author contributions}

W.G., L.H., L.T., P.S. conceived and initiated the study. L.T. and Z.Z. performed the genome sequencing and bioinformatics analyses. P.S. and L.T. performed most of the experiments and L.G., J.W., T.H., J.Z., Y. Zhang, Y. Zhao, Y. Liu, Y.S., Y.T., Y. Lu, and J.Y. assisted in part of the experiment. L.T., P.S., and Z.Z. wrote the manuscript. C.X., M.J., R.J.P., W.G., and L.H. revised the manuscript.

\section{Competing interests}

The authors declare no competing interests. 


\section{Additional information}

Supplementary information is available for this paper at https://doi.org/10.1038/s41467020-14776-1.

Correspondence and requests for materials should be addressed to L.H. or W.G.

Peer review information Nature Communications thanks Amit Rai, Gane Wong, Philipp Zerbe and the other, anonymous, reviewer(s) for their contribution to the peer review of this work. Peer reviewer reports are available.

Reprints and permission information is available at http://www.nature.com/reprints

Publisher's note Springer Nature remains neutral with regard to jurisdictional claims in published maps and institutional affiliations. (c) (i) Open Access This article is licensed under a Creative Commons Attribution 4.0 International License, which permits use, sharing, adaptation, distribution and reproduction in any medium or format, as long as you give appropriate credit to the original author(s) and the source, provide a link to the Creative Commons license, and indicate if changes were made. The images or other third party material in this article are included in the article's Creative Commons license, unless indicated otherwise in a credit line to the material. If material is not included in the article's Creative Commons license and your intended use is not permitted by statutory regulation or exceeds the permitted use, you will need to obtain permission directly from the copyright holder. To view a copy of this license, visit http://creativecommons.org/ licenses/by/4.0/.

(C) The Author(s) 2020 